\title{
The Singularity Threshold of the Nonlinear Sigma Model Using 3D Adaptive Mesh Refinement
}

\author{
Steven L. Liebling \\ Theoretical and Computational Studies Group \\ Southampton College, Long Island University \\ Southampton, NY 11968
}

(Dated: October 24, 2018)

\begin{abstract}
Numerical solutions to the nonlinear sigma model (NLSM), a wave map from $3+1$ Minkowski space to $S^{3}$, are computed in three spatial dimensions (3D) using adaptive mesh refinement (AMR). For initial data with compact support the model is known to have two regimes, one in which regular initial data forms a singularity and another in which the energy is dispersed to infinity. The transition between these regimes has been shown in spherical symmetry to demonstrate threshold behavior similar to that between black hole formation and dispersal in gravitating theories. Here, I generalize the result by removing the assumption of spherical symmetry. The evolutions suggest that the spherically symmetric critical solution remains an intermediate attractor separating the two end states.
\end{abstract}

Nonlinear sigma models have attracted the attention both of physicists and mathematicians. For physicists, the models represent the possibility of describing particles with a field theory, while mathematicians study singularity formation in geometrically motived nonlinear models. Recently, a nonlinear sigma model has attracted the attention of relativists because it demonstrates behavior similar to black hole critical phenomena discovered by Choptuik [1].

Studying the gravitational collapse of a spherically symmetric scalar field, he found that initial data with large energy formed a black hole while that for small initial energy dispersed its energy to infinity. By tuning the initial energy, he approached the so-called critical regime which separates these two end states. In this regime, solutions approach a unique, universal solution which demonstrates self-similarity.

The model studied here serves as a useful toy model for such threshold behavior because it also possesses two stable end-states, dispersal and singularity formation. These end-states serve as analogues for the gravitating case in which initial data can either disperse or form a black hole. Similar to Choptuik's gravitating model, spherically symmetric studies of this model have found a universal, self-similar critical solution [2, 3]. The utility of the nonlinear sigma model arises from its relative simplicity compared to any of a variety of gravitating models. Perhaps the study of such nonlinear, flat systems will guide the way to further insight in the gravitating models.

While questions about the nature of black hole critical behavior remain, many unexplored "experiments" have yet to be conducted. While many matter models have been studied (for a review see 顿), generally these studies have been restricted to spherical symmetry. The only work to model fully nonlinear collapse in less symmetry is that of Abrahams and Evans [5] who studied vacuum gravitational collapse in axisymmetry. One rea- son for the scarcity of multi-dimensional evolutions of the critical regime is the extreme resolution demanded by approach to scale invariance. Perturbation methods are a complementary approach with recent work [6, 7] suggesting that spherical critical solutions in the scalar field and perfect fluid cases extend to the nonspherical regime. While work is underway to duplicate and extend the work of Abrahams and Evans in axisymmetry [8], it makes sense to look ahead to a simple model in three spatial dimensions.

To obtain the resolution required for evolving selfsimilar solutions, Choptuik developed a computational infrastructure in one spatial dimension which dynamically and locally adds numerical resolution where needed using adaptive mesh refinement (AMR). With this infrastructure, fine subgrids are added and subtracted to the computational domain providing resolution only where and when needed. Because the computational cost scales as a power law in the spatial dimension (i.e. doubling the resolution of a $d$-dimensional evolution requires a factor $2^{d+1}$ more work), it is expected that AMR will be absolutely crucial in higher dimensions for well-resolved evolutions of interest, in particular black hole critical phenomena or black hole collisions.

Constructing such a gravitating model with AMR is an ambitious project. Instead I report on the construction of a 3D AMR code of the nonlinear sigma model, which genuinely requires AMR and holds physical interest.

The NLSM Model: The nonlinear sigma model studied here represents a mapping from the base space of $3+1$ Minkowski to a target space of $S^{3}$. In spherical symmetry, it is common to choose the hedgehog ansatz for the map reducing the dynamics to that of a single spherically symmetric field $\chi(r, t)$. Here a simple gener- 
alization of this ansatz is chosen

$$
\phi^{a}=\left(\begin{array}{c}
\sin \chi(x, y, z, t) \sin \theta \sin \varphi \\
\sin \chi(x, y, z, t) \sin \theta \cos \varphi \\
\sin \chi(x, y, z, t) \cos \theta \\
\cos \chi(x, y, z, t)
\end{array}\right)
$$

where $\theta$ and $\varphi$ are the usual spatial angles. The dynamics reduce to the scalar field $\chi(x, y, z, t)$ which satisfies the equation of motion

$$
\ddot{\chi}=\chi_{, x x}+\chi_{, y y}+\chi_{, z z}-\frac{\sin 2 \chi}{r^{2}},
$$

where commas indicate partial differentiation with respect to subscripted coordinates, an overdot denotes $\partial / \partial t$, and $r \equiv \sqrt{x^{2}+y^{2}+z^{2}}$. The equation of motion (2) implies the regularity condition $\chi(0,0,0, t)=0$ which is enforced by the evolution procedure. This ansatz requires that the origin is singled out as a special point. More elegant generalizations may be considered in the future.

The energy density of the map is given by

$$
\rho=\frac{1}{2}\left[(\dot{\chi})^{2}+\left(\chi_{, x}\right)^{2}+\left(\chi_{, y}\right)^{2}+\left(\chi_{, z}\right)^{2}\right]+\frac{\sin ^{2} \chi}{r^{2}} .
$$

The angular momentum densities are given by [9]

$$
M^{\mu \nu}=\int d^{3} x\left(T^{0 \mu} x^{\nu}-T^{0 \nu} x^{\mu}\right),
$$

so that the $z$-component of the angular momentum, for example, is

$$
J_{z}=\int d^{3} x M^{x y}=\int d^{3} x \dot{\chi}\left(y \chi_{, x}-x \chi_{, y}\right) .
$$

While the map allows for the possibility of a texture charge associated with the third homotopy group, only initial data with zero charge is considered here. The model requires initial data $\chi(x, y, z, 0)$ and $\dot{\chi}(x, y, z, 0)$ be specified at the initial time.

Various types of initial data have been implemented and are described in Table I. Some of these families are defined in terms of a generalized Gaussian pulse defined by

$$
G(x, y, z)=A e^{-(\tilde{r}-R)^{2} / \delta^{2}}
$$

where $\tilde{r}$ is a generalized radial coordinate

$$
\tilde{r}=\sqrt{\epsilon_{x}\left(x-x_{c}\right)^{2}+\epsilon_{y}\left(y-y_{c}\right)^{2}+\left(z-z_{c}\right)^{2}} .
$$

Such a pulse depends on parameters: amplitude $A$, shell radius $R$, pulse width $\delta$, pulse center $\left(x_{c}, y_{c}, z_{c}\right)$, and skewing factors $\epsilon_{x}$ and $\epsilon_{y}$. For $\epsilon_{x} \neq 1 \neq \epsilon_{y}$ such a pulse has elliptic cross section. Family (a) represents a single pulse for which the parameter $\nu$ takes the values $\{-1,0,+1\}$ for an approximately out-going, timesymmetric, or approximately in-going pulse. The angular

\begin{tabular}{|c|l|c|c|}
\hline & Description & $\chi(x, y, z, 0)$ & $\dot{\chi}(x, y, z, 0)$ \\
\hline \hline a & Ellipsoid & $G$ & $\begin{array}{c}\nu \frac{\partial G}{\partial \tilde{r}} \\
+\Omega_{z}\left(y G_{, x}-x G_{, y}\right)\end{array}$ \\
\hline b & Two pulses & $G_{1}+G_{2}$ & $v_{1} \frac{\partial G_{1}}{\partial x}+v_{2} \frac{\partial G_{2}}{\partial x}$ \\
\hline $\mathrm{c}$ & Toroid & $A e^{-z^{2} / \delta^{2}} e^{-\left(\epsilon_{x} x^{2}+\epsilon_{y} y^{2}\right)^{2} / \delta^{2}}$ & 0 \\
\hline
\end{tabular}

TABLE I: List of various initial data families. For families (a)-(c) both the field $\chi(x, y, z, 0)$ and its time derivative $\dot{\chi}(x, y, z, 0)$ are shown in terms of various parameters. The terms $G, G_{1}$, and $G_{2}$ represent unique Gaussian pulses as defined in Eq. (6). In family (b), the parameters $v_{1}$ and $v_{2}$ are the respective velocities of the two pulses, generally chosen to have a grazing collision.

momentum of the pulse about the $z$-axis is proportional to the parameter $\Omega_{z}$ as well as to $\left(\epsilon_{x}-\epsilon_{y}\right)^{2}$.

Numerical method: RNPL [10] is used to develop and debug a stable and convergent unigrid code which solves Eq. (2) using finite differences and iterative CrankNicholson. The RNPL generated update procedure is called from the AMR code to evolve any given fine grid. The AMR implementation follows that of Berger and Oliger [11] with some simplifications:

(1) Instead of estimating truncation error, I use the energy density as a criterion of refinement. In particular, normalizing the energy density $\rho$ by the grid resolution $1 / h$, the refinement criterion is $h^{2} \rho>\epsilon$, where $\epsilon$ is a userspecified threshold. This simplification is less general than truncation error but provides an easily computable and smooth function from which to estimate resolution requirements.

(2) Fine grids are completely contained within their parents. Grids do not overlap or abut other grids at the same level. This restriction reduces the maximum obtainable efficiency but provides for considerable simplification.

(3) Fine grids are created strictly aligned with parent grids with no rotation. The transformations necessary to relax this would be especially onerous in the gravitating case.

(4) The ratio of refinement between parent and child grids is constrained to be an even integer.

The first two of these restrictions appear especially suited for the case of central collapse studied here, and the intention is to relax them for more general problems.

The usefulness of AMR depends obviously on whether it produces correct solutions and whether it allows for high resolution with only proportional work. Starting with the unigrid code, convergence and energy conservation were confirmed. The AMR results were then checked against high resolution unigrid results. Perhaps a stronger test is that this code finds the same results for spherically symmetric initial data as that in [2] (as demonstrated in Fig. 1). As an example of the benefit of AMR consider Fig. 2. A rough estimate of the com- 


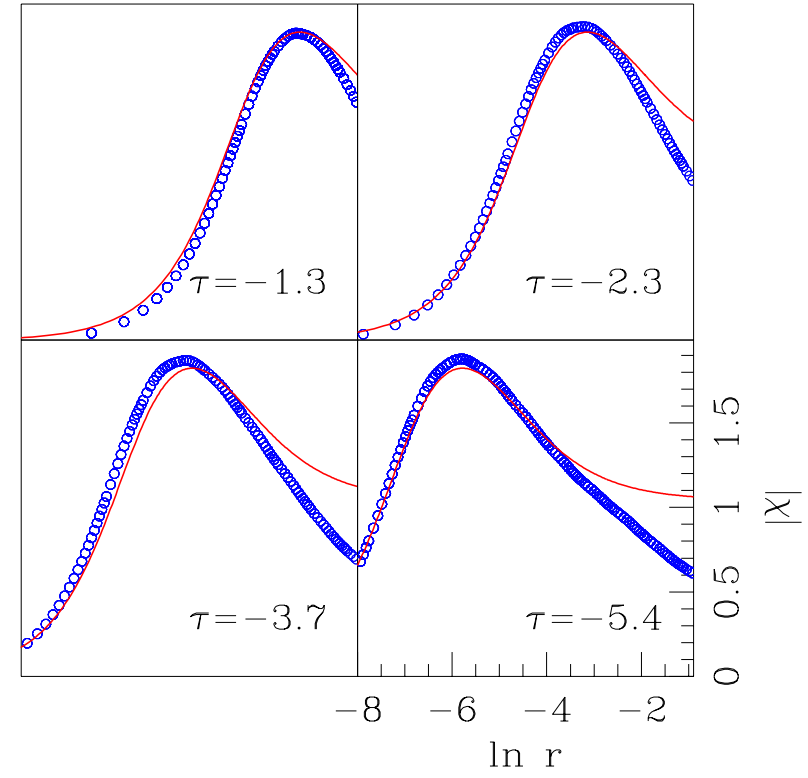

FIG. 1: Approach of near-critical evolution to self similarity. The numerical evolution is shown (circles) for spherically symmetric initial data at times near the collapse time. The initial data is a member of family (a) in Table with $\epsilon_{x}=\epsilon_{y}=1$ and $\Omega_{z}=0$. The data represents a $(x>0, y=0, z=0)$ cut with every point shown. The excited $n=1$ self-similar solution is shown (solid line) with $\tau \equiv \ln \left|T^{*}-T\right|$ where $T^{*}$ is the time of collapse (so that collapse occurs at $\tau \rightarrow-\infty$ ). The collapse time of the $n=1$ solution is chosen so that the two solutions coincide for the first frame only. That they coincide for the other frames indicates the approach to the self-similar solution.

putational work for the entire evolution is $2^{8}$, in units where the work for a unigrid evolution at the coarsest resolution is unity. In comparison, to achieve uniform resolution equal to that of the finest sub-grid would require work equal to $\left(2^{9}\right)^{3+1}$, a factor $2^{26}$ more work than that with AMR.

Results: As discussed in [2], solutions generically evolve to one of two stable end states. For large initial data, the energy density collapses toward the origin suggesting the formation of a singularity. As in the strictly spherical case, the usual caveat applies that we only have indications, not proof, that a singularity is forming. For small initial data, the energy density implodes through the origin and then disperses to infinity. Removing the restriction to spherical symmetry has not revealed any additional stable end states.

The aim is to investigate the region of solution space separating these two end states. In the jargon of nonlinear dynamics, we look at the so-called critical surface occurring between the two basins of attraction. To do so, one chooses a 1-parameter family of initial data with the property that data with small parameter disperses while large parameter initial data forms a singularity. By tuning the free parameter according to the resulting end
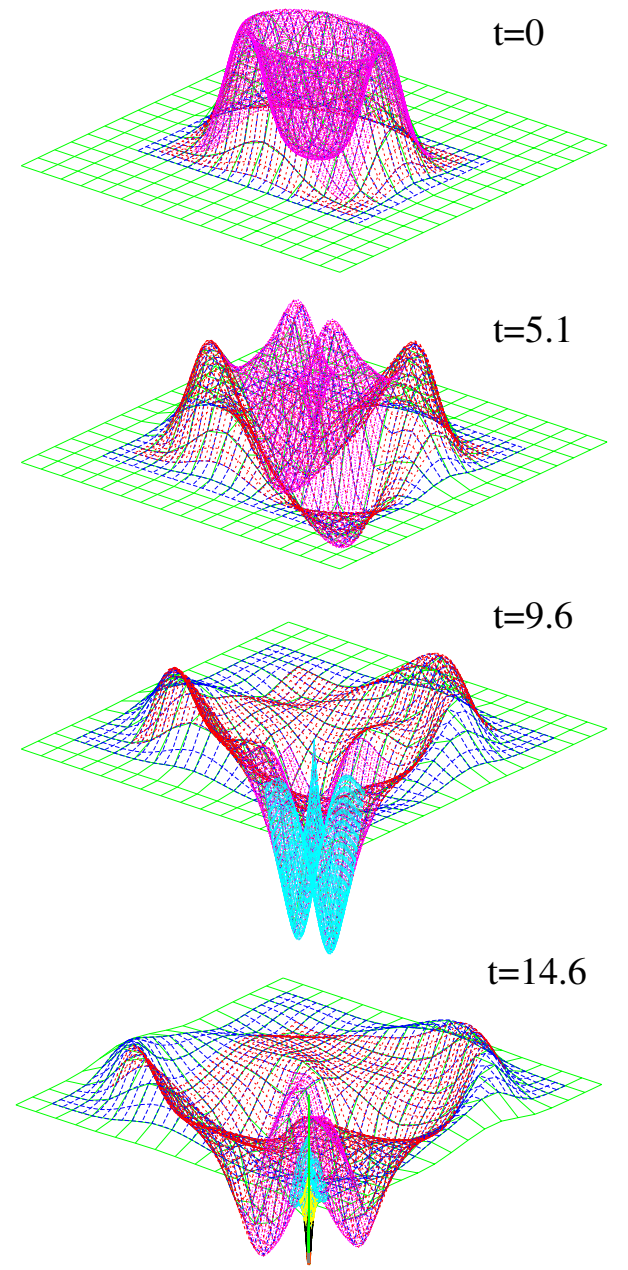

FIG. 2: Snapshots of slightly sub-critical evolution. The field $\chi(t, x, y, z=0)$ is shown at four times for a family of initial data with angular momentum about the $z$-axis. For clarity, only every other point in the $x$ and $y$ directions is shown (every fourth point). At the final time, all ten grids are shown with a refinement factor of 2 . Greater detail of this last frame is shown in Fig. 3. The initial data is a member of family (a) from Table with parameters $A \approx 1.3359, R=8$, $\delta=3, x_{c}=y_{c}=z_{c}=0, \epsilon_{x}=0.5, \epsilon_{y}=1, \nu=0$, and $\Omega_{z}=0.4$. The dimensionless ratio of the angular momentum to the energy squared is $J / E^{2}=0.0025$.

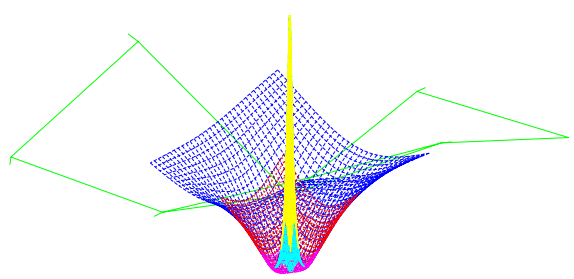

FIG. 3: Detail of the peak region from the final frame of Fig. 2. Only the finest four grids are shown (every point is shown) along with the coarsest grid in order to compare the resolution achieved with respect to the coarse grid. The finest grid has a resolution a factor of $2^{9}$ finer than the coarse grid. 
state of the evolution, the critical surface is approached.

The previous results in spherical symmetry indicated that all initial data of compact support, when tuned, approached the same universal, self-similar solution [2]. This critical solution happens to be one of a family of solutions found by Aminneborg and Bergstrom by assuming self-similarity and solving the resulting ODE [12]. A linear perturbation analysis revealed the $n=1$ solution to have the requisite single unstable mode necessary to be a proper critical solution (a so-called intermediate attractor).

This solution is shown in coordinates adapted to its self-similarity in Fig. 1. Given a self-similar solution which takes the form $f(r, t)=f\left(r /\left|T^{*}-T\right|\right)$, one can recast it as $g\left(\ln r-\ln \left|T^{*}-T\right|\right)$. This form makes apparent that the solution executes linear motion in log space and log time, where one is free to set the collapse time $T^{*}$. Thus, in Fig. 目 the self-similar solution travels leftward retaining its shape.

Along with the $n=1$ solution is shown a cut along the $x$-axis of a near-critical evolution from spherically symmetric initial data. Such an evolution would be expected to agree with previous results in spherical symmetry, as it appears to do in the plot. The collapse time of the $n=1$ solution was chosen so that the two solutions agree in the first frame. The $n=1$ solution is then completely determined at other times by translation in $\ln r$.

It was also observed in [2] that whenever the range of $\chi$ exceeded $\pi$ at a given time, a singularity would eventually form in its future. However, evolutions of non-spherically symmetric families do not obey such a condition, having exceeded $\pi$ while remaining nonsingular. One still finds solutions which disperse (sub-critical) separated from those which form a singularity (supercritical).

For such non-symmetric initial data, the question is whether some different critical solution appears. If the $n=1$ solution has non-symmetric unstable modes not present in spherical symmetry, then a change in critical solution would be expected. However, all such nonsymmetric variations of the types of initial data in Table have failed to find a different critical solution.

An example from initial data with angular momentum is shown in Fig. 2. The pulse evolves in a nontrivial manner eventually dispersing much of its energy toward the edges of the grid. However, near the origin, a spherically symmetric waveform emerges which propagates toward the origin. In this case, the waveform has negative amplitude (the model is invariant with respect to the transformation $\chi \rightarrow-\chi)$. Further detail of this waveform is shown in Fig. 3. The upward sweep at the origin corresponds to the regularity constraint mentioned previously that $\chi(0,0,0, t)=0$.

Another example of initial data with angular momentum is shown in Fig. 同. Two pulses are sent towards one another and, for sufficiently large initial amplitude, sin-

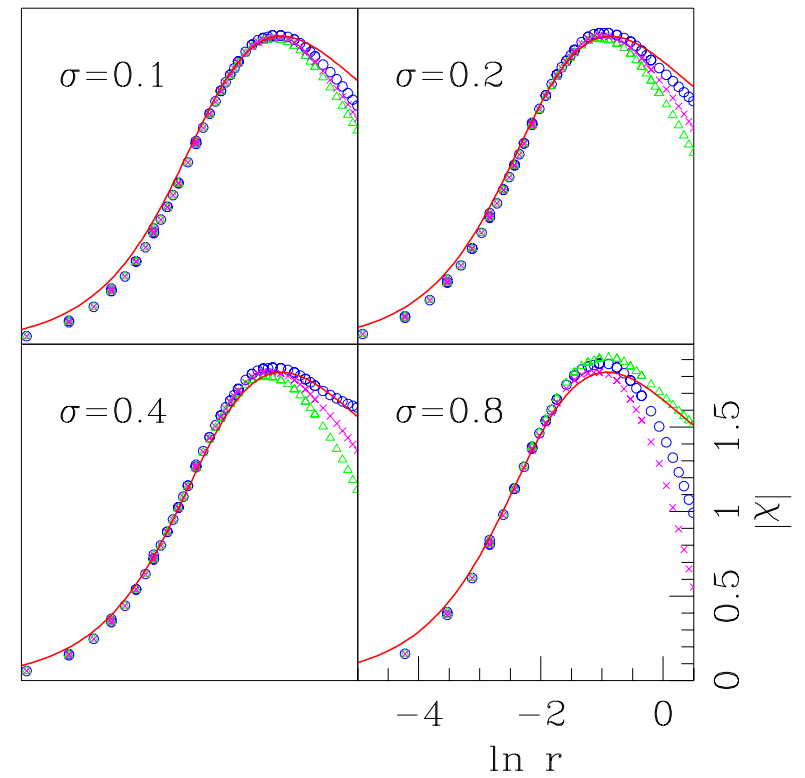

FIG. 4: Demonstration of approach to a spherically symmetric solution at criticality. Initially asymmetric initial data is tuned and slightly sub-critical solutions are shown above for different families. Here $\sigma$ is a measure of the asymmetry ( $\sigma=0$ corresponds to spherical symmetry) which partially defines family (a) in Table fivia $\epsilon_{x}=1+\sigma$ and $\epsilon_{y}=1-\sigma$. For each value of $\sigma$, three slices are shown: $(x>0,0,0)$ (circles), $(0, y>0,0)$ (triangles), and $(0,0, z>0)$ (crosses). Their general agreement near the origin demonstrates approach to spherical symmetry. Also shown (line) is the $n=1$ self-similar solution (with collapse time adjusted to match the solution).

gularity formation occurs. Four frames of a near critical evolution show the region near the origin approaching the spherically symmetric, self-similar solution.

A more systematic exploration away from spherical symmetry is shown in Fig. 4 . Here, $\sigma$ parameterizes the asymmetry of the initial data, and near-critical solutions are shown for four different values of $\sigma$. Furthermore, for each case the region near the origin (the region which approaches self-similarity) is shown along the three different axes. Their agreement suggests that this region approaches spherical symmetry.

There remains, however, the possibility that some other spherically symmetric self-similar solution becomes the critical solution away from spherical symmetry. Though there is no evidence of any transition, the various excited self-similar solutions of 12 are very similar near the origin consisting of a peak followed by oscillations about $\chi=\pi / 2$. To distinguish among them, a perturbation analysis allowing for non-symmetric modes would be appropriate, with the critical solution being the one with a single unstable mode.

The evidence therefore suggests that the $n=1$ selfsimilar solution remains the intermediate attractor away 


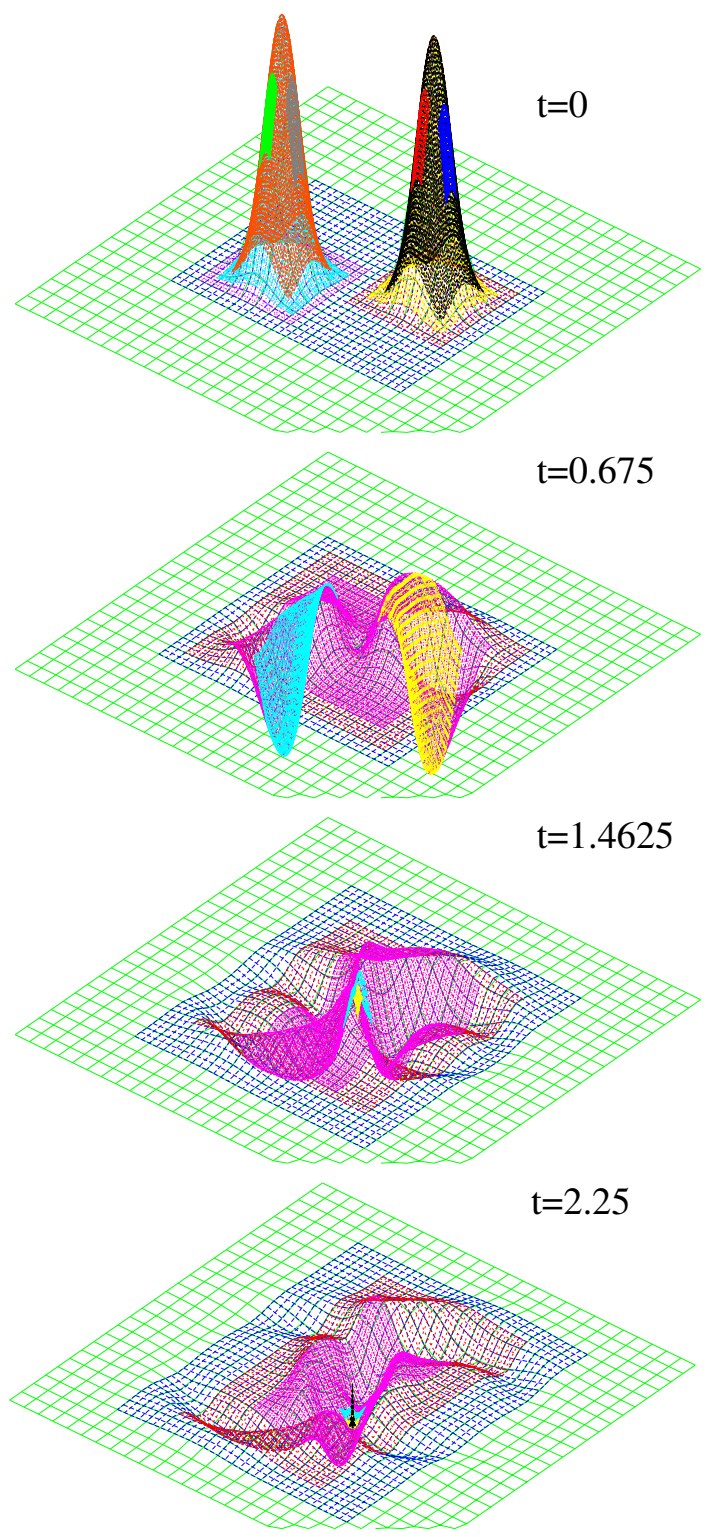

FIG. 5: Snapshots of a slightly sub-critical collision of two grazing pulses. The field $\chi(t, x, y, z=0)$ is shown in the $x-$ $y$ plane at four times for two pulses with linear momentum in $\pm x$ directions. At late times, the region near the origin (the fine grids in the center of the final two frames) rapidly approaches spherical symmetry and self-similarity. The initial data consists of family (b) from Table f with parameters $A_{1}=$ $A_{2}=11.5, R_{1}=R_{2}=0, \delta_{1}=\delta_{2}=0.5,\left(x_{c 1}, y_{c 1}, z_{c 1}\right)=$ $\left(-x_{c 2},-y_{c 2}, z_{c 2}\right)=(1.4,0.7,0), \epsilon_{x 1}=\epsilon_{x 2}=1, \epsilon_{y 1}=\epsilon_{y 2}=1$, and $v_{1}=-v_{2}=1$. The dimensionless ratio of the angular momentum to the energy squared is $J / E^{2}=0.0008$. from spherical symmetry, but a perturbation analysis should settle the issue. Further work will likely be directed towards (1) using the AMR infrastructure with a gravitating model, (2) relaxing the noted simplifications in the clustering, and (3) distributing the grids using MPI along the lines of [13].

Acknowledgments: Many thanks to Frans Pretorius and Matthew W. Choptuik for providing me with the extremely useful Data-Vault, a software application they are developing for visualizing AMR data. Thanks go also to Richard A. Matzner for reviewing the manuscript. I appreciate the support of NSF PHY-9900644 and of the financial support of Southampton College.

[1] M. W. Choptuik, Phys. Rev. Lett. 70, 9 (1993).

[2] S. L. Liebling, E. W. Hirschmann, and J. Isenberg, J. Math. Phys. 41, 5691 (2000), math-ph/9911020.

[3] P. Bizoń, T. Chmaj, and Z. Tabor, Nonlinearity 13, 1411 (2000).

[4] C. Gundlach, Living Rev. Rel. 2, 4 (1999), grqc/0001046.

[5] A. M. Abrahams and C. R. Evans, Phys. Rev. Lett. 70, 2980 (1993).

[6] C. Gundlach, Phys. Rev. D65, 084021 (2002), grqc/9906124.

[7] J. M. Martin-Garcia and C. Gundlach, Phys. Rev. D59, 064031 (1999), gr-qc/9809059.

[8] M. W. Choptuik, E. W. Hirschmann, S. L. Liebling, and F. Pretorius, In preparation (2002).

[9] L. H. Ryder, Quantum Field Theory (Cambridge University Press, 1996).

[10] M. W. Choptuik and R. L. Marsa, Rapid numerical prototyping language (2002), Software available from: ftp://laplace.physics.ubc.ca/pub/rnpl.

[11] M. J. Berger and J. Oliger, J. Comp. Phys. 53, 484 (1984).

[12] S. Aminneborg and L. Bergstrom, Phys. Lett. B362, 39 (1995), astro-ph/9511064.

[13] S. D. Hern, Ph.D. thesis (1999), gr-qc/0004036. 